\title{
Comparison of two projection methods for the solution of frictional contact problems
}

Shougui Zhang ${ }^{1 *}$ (D) and Ruisheng Ran ${ }^{2}$

"Correspondence:

shgzhang@cqnu.edu.cn

'School of Mathematical Sciences, Chongqing Normal University,

Chongqing, P.R. China

Full list of author information is

available at the end of the article

\section{空 Springer}

\begin{abstract}
Frictional contact problems in linear elasticity are considered in this paper. The contact constraint is imposed in the weak sense using the fixed point method, which leads to a variational equation problem. For solving such a nonlinear variational problem, we study two projection methods using different self-adaptive rules. Based on the self-adaptive projection method, we propose a modified self-adaptive rule which is more effective to update the parameter. The methods can be implemented easily in conjunction with the boundary element method for the solution. Numerical experiments are reported to illustrate theoretical results.
\end{abstract}

Keywords: Frictional contact problem; Variational inequality; Projection method; Self-adaptive rule; Boundary element

\section{Introduction}

Frictional contact phenomena among deformable bodies or between deformable and rigid bodies abound in industry and daily life; they play an important role in many fields of solid mechanics $[1,2]$. Because of the nonlinearity, the exact solution is difficult to be obtained. Therefore, a considerable effort has been made in modeling and numerical simulations of contact processes. Most of the time, the mathematical formulation of the frictional contact problem is reformulated as a minimization problem or a variational inequality of the second kind. Theory of contact problems and their numerical approximations has been extensively developed during the past decades [3-10]. Among the most popular methods we mention semi-smooth Newton methods and projection methods. Although the semismooth Newton method is well known as the local superlinear convergence for nonlinear problems, this method converges fast only if the penalty parameter of this method is big enough, which may result in a badly conditioned problem; while the projection method with a fixed penalty parameter will be depredated significantly if the parameter is either too small or too large. Therefore, the convergence speed of these methods is sensitive to the choice of parameters. In this paper, we study the numerical solutions of frictional contact problems using the projection method with two self-adaptive rules for the parameter.

The first method, called the self-adaptive projection method in this paper, is applicable for solving monotone variational inequalities of the first kind $[9,11-13]$. Using the equivalence between the frictional contact problem and a variational formulation with a projection fixed point problem [14-16], our method formulates the contact boundary

(c) The Author(s) 2019. This article is distributed under the terms of the Creative Commons Attribution 4.0 International License (http://creativecommons.org/licenses/by/4.0/), which permits unrestricted use, distribution, and reproduction in any medium, provided you give appropriate credit to the original author(s) and the source, provide a link to the Creative Commons license, and indicate if changes were made. 
condition into a sequence of Robin boundary conditions. We prove the unconditional convergence in function spaces. The main advantage of the method is that, as compared with other methods, there is a combination of the projection method and the self-adaptive rule which uses iterative functions to update the parameter automatically.

The second method, called the modified self-adaptive projection method in this paper, is based on the self-adaptive projection method. This method uses a modified selfadaptive rule to better adjust the parameter and accelerate the convergence speed of the method, for any initial function. In addition, we note that the proof of the self-adaptive projection method for frictional contact problems can be easily extended to the modified self-adaptive projection method as well.

In these two methods, the key unknowns of frictional contact problems are displacement and stress on the contact boundary, which are considered primary variables and can be related in a linear system by the boundary element method (BEM) $[9,10,17,18]$. Besides, the BEM significantly reduces expense mesh generation because the formulation of the problem is concluded to the boundary of the domain. Therefore the BEM is a natural numerical tool for the solution of frictional contact problems. In this paper, we apply projection methods combined with the BEM for the numerical solution of frictional contact problems.

The rest of the paper is organized as follows. First, we introduce the variational formulation of the classical frictional contact problem via a projection fixed point problem. Section 3 presents the self-adaptive projection method and the convergence analysis. Section 4 describes the implementation detail of the two self-adaptive rules and the boundary element approximation for the method. In Sect. 5, we give some numerical results that confirm our theoretical findings. In particular, we show that the modified self-adaptive projection method has better convergence speed and stability for all parameters. Finally, Sect. 6 concludes the paper with some remarks.

\section{Setting of the problem}

We consider the classical frictional contact problem with a rigid foundation. Let $\Omega$ be an open and bounded domain in $\mathbb{R}^{2}$, with a Lipschitz boundary $\Gamma=\partial \Omega$. The boundary $\Gamma$ is partitioned as three mutually disjoint parts $\Gamma_{D}, \Gamma_{N}$, and $\Gamma_{C} \neq \varnothing$, where Dirichlet, Neumann, and frictional contact boundary conditions are prescribed. For simplicity, we assume that there are no volume forces acting on the body. We use $\mathbf{n}$ and $\mathbf{t}$ to denote the outward normal and tangential vectors of $\Gamma$, respectively. For given boundary traction $\hat{\mathbf{t}} \in$ $\left(L^{2}\left(\Gamma_{N}\right)\right)^{2}$ and obstacle $g \in L^{2}\left(\Gamma_{C}\right)$ with $g>0$, the problem is to determine the displacement u such that

$$
\left\{\begin{array}{l}
\operatorname{div} \boldsymbol{\sigma}(\mathbf{u})=\mathbf{0} \text { in } \Omega \\
\mathbf{u}=\mathbf{0} \text { on } \Gamma_{D} \\
\boldsymbol{\sigma}(\mathbf{u}) \mathbf{n}=\hat{\boldsymbol{t}} \text { on } \Gamma_{N} \\
u_{n}=0 \text { on } \Gamma_{C}
\end{array}\right.
$$

and the following friction condition on $\Gamma_{C}$

$$
\left\{\begin{array}{l}
\text { if } \mathbf{u}_{t}=\mathbf{0} \text { then }\left|\boldsymbol{\sigma}_{t}(\mathbf{u})\right| \leq g \\
\text { if } \mathbf{u}_{t} \neq \mathbf{0} \text { then } \boldsymbol{\sigma}_{t}(\mathbf{u})=-g \frac{\mathbf{u}_{t}}{\left|\mathbf{u}_{t}\right|},
\end{array}\right.
$$


where $\boldsymbol{u}_{t}$ and $\sigma_{t}$ are the tangential contact displacement and the tangential contact traction, respectively. In this paper, we adopt the following decomposition for the displacement and the stress vector fields:

$$
\mathbf{u}=u_{n} \mathbf{n}+\mathbf{u}_{t} \quad \text { and } \quad \boldsymbol{\sigma}(\mathbf{u}) \mathbf{n}=\sigma_{n}(\mathbf{u}) \mathbf{n}+\boldsymbol{\sigma}_{t}(\mathbf{u})
$$

Let us introduce the following Hilbert space:

$$
\boldsymbol{V}:=\left\{\mathbf{v} \in\left(H^{1}(\Omega)\right)^{2},\left.\mathbf{v}\right|_{\Gamma_{D}}=\mathbf{0},\left.u_{n}\right|_{\Gamma_{C}}=0\right\}
$$

and notations

$$
\begin{aligned}
& a(\mathbf{u}, \mathbf{v}):=\int_{\Omega} \boldsymbol{\sigma}(\mathbf{u}): \boldsymbol{\epsilon}(\mathbf{v}) d x, \\
& j(\mathbf{v}):=\int_{\Gamma_{C}} g\left|\mathbf{v}_{t}\right| d s_{x}, \\
& L(\mathbf{v}):=\int_{\Gamma_{N}} \hat{\mathbf{t}}(x) \cdot \mathbf{v}(x) d s_{x} .
\end{aligned}
$$

Problem (2.1)-(2.6) is then equivalent to the following variational inequality of the second kind:

$$
\left\{\begin{array}{l}
\text { Find } \mathbf{u} \in \boldsymbol{V} \text { satisfying } \\
a(\mathbf{u}, \mathbf{v}-\mathbf{u})+j(\mathbf{u})-j(\mathbf{v}) \geq L(\mathbf{v}-\mathbf{u}), \quad \forall \mathbf{v} \in \boldsymbol{V}
\end{array}\right.
$$

or a convex minimization problem

$$
\left\{\begin{array}{l}
\text { Find } \mathbf{u} \in \boldsymbol{V} \text { such that } \\
J(\mathbf{u})=\min _{\mathbf{v} \in \mathbf{V}} J(\mathbf{v}):=\frac{1}{2} a(\mathbf{v}, \mathbf{v})-L(\mathbf{v})+j(\mathbf{v}) .
\end{array}\right.
$$

It follows from the theory of variational inequalities that problem (2.7), or equivalently (2.8), admits a unique solution [1,2]. Let us introduce a projection notation $[\mathbf{x}]_{\alpha}$ for $\alpha \in \mathbb{R}^{+}$ and vector $\mathbf{x} \in \mathbb{R}^{2}$

$$
[\mathbf{x}]_{\alpha}:= \begin{cases}\mathbf{x} & \text { if }|\mathbf{x}| \leq \alpha \\ \alpha \frac{\mathbf{x}}{|\mathbf{x}|} & \text { otherwise }\end{cases}
$$

Then we have the next result for the frictional boundary condition, which has been pointed out in [15].

Lemma 2.1 For all $\rho>0$, the frictional contact condition (2.5)-(2.6) on $\Gamma_{C}$ is equivalent to

$$
\boldsymbol{\sigma}_{t}(\mathbf{u})+\left[\rho \mathbf{u}_{t}-\boldsymbol{\sigma}_{t}(\mathbf{u})\right]_{g}=\mathbf{0} \text { on } \Gamma_{C}
$$


Now, we define the following residual function:

$$
\boldsymbol{R}_{\rho}\left(\boldsymbol{u}_{t}, \boldsymbol{\sigma}_{t}\right)=\boldsymbol{\sigma}_{t}+\left[\rho \boldsymbol{u}_{t}-\boldsymbol{\sigma}_{t}\right]_{g} \quad \text { on } \Gamma_{C} .
$$

As a result, the nonsmooth projection equation (2.9) can be rewritten as $\boldsymbol{R}_{\rho}\left(\boldsymbol{u}_{t}, \boldsymbol{\sigma}_{t}\right)=\mathbf{0}$. Then, we propose a new equivalent formulation for the frictional boundary condition (2.5)-(2.6). For given $\omega \neq 0, \boldsymbol{R}_{\rho}\left(\boldsymbol{u}_{t}, \boldsymbol{\sigma}_{t}\right)=\mathbf{0}$ can be written in the following form:

$$
\boldsymbol{\sigma}_{t}+\rho \boldsymbol{u}_{t}=\boldsymbol{\sigma}_{t}+\rho \boldsymbol{u}_{t}-\omega \boldsymbol{R}_{\rho}\left(\boldsymbol{u}_{t}, \boldsymbol{\sigma}_{t}\right)
$$

Next, we apply the Green formula to (2.1)-(2.6) and obtain the variational formulation

$$
a(\boldsymbol{u}, \boldsymbol{v})-\int_{\Gamma_{C}} \boldsymbol{\sigma}_{t} \cdot \boldsymbol{v}_{t} d s_{x}=L(\boldsymbol{v}) \quad \forall \boldsymbol{v} \in \boldsymbol{V} .
$$

Consequently, we obtain the following variational and projection formulations for the frictional contact problem (2.1)-(2.6):

$$
\left\{\begin{array}{l}
a(\boldsymbol{u}, \boldsymbol{v})-\int_{\Gamma_{C}} \boldsymbol{\sigma}_{t} \cdot \boldsymbol{v}_{t} d s_{x}=L(\boldsymbol{v}) \quad \forall \boldsymbol{v} \in \boldsymbol{V}, \\
\boldsymbol{\sigma}_{t}+\rho \boldsymbol{u}_{t}=\boldsymbol{\sigma}_{t}+\rho \boldsymbol{u}_{t}-\omega \boldsymbol{R}_{\rho}\left(\boldsymbol{u}_{t}, \boldsymbol{\sigma}_{t}\right) \quad \text { on } \Gamma_{C} .
\end{array}\right.
$$

Using this equivalent formulation, we can suggest a self-adaptive projection method for the frictional contact problem in the next section.

\section{Self-adaptive projection method}

In order to solve problem (2.13), we rewrite (2.11) as the following Robin iterative scheme as in [9-13]:

$$
\boldsymbol{\sigma}_{t}^{(k+1)}+\rho \boldsymbol{u}_{t}^{(k+1)}=\boldsymbol{\sigma}_{t}^{(k)}+\rho \boldsymbol{u}_{t}^{(k)}-\omega \boldsymbol{R}_{\rho}\left(\boldsymbol{u}_{t}^{(k)}, \boldsymbol{\sigma}_{t}^{(k)}\right) \quad \text { on } \Gamma_{C} .
$$

Then we obtain the projection method for the numerical solution of the frictional contact problem. In this method, there are two parameters $\omega \in(0,2)$ and $\rho>0$ which affect the convergence speed. We note that the good parameter $\omega$ should be less than and close to 2 [9-11]. Although the method converges for any fixed parameter $\rho>0$, the efficiency of the method depends on the parameter $\rho$ heavily.

Here, we propose a projection method with a self-adaptive variable sequence of parameters $\left\{\rho_{k}\right\}[9,10]$. In the following we need a nonnegative sequence $\left\{s_{k}\right\}$ satisfying

$$
\sum_{k=0}^{+\infty} s_{k}<+\infty
$$

Now, we present the following self-adaptive projection method for the frictional contact problem.

\section{Algorithm 1}

Step 0: Choose initial functions $\boldsymbol{u}_{t}^{(0)} \in\left(H^{1 / 2}\left(\Gamma_{C}\right)\right)^{2}, \boldsymbol{\sigma}_{t}^{(0)} \in\left(L^{2}\left(\Gamma_{C}\right)\right)^{2}, \rho \in \mathbb{R}^{+}$, and $\omega \in(0,2)$; set $\rho_{0}=\rho$ and $k:=0$. 
Step 1: Compute $\boldsymbol{R}_{\rho_{k}}\left(\boldsymbol{u}_{t}^{(k)}, \boldsymbol{\sigma}_{t}^{(k)}\right)$ according to (2.10).

Step 2: Find $\left(\boldsymbol{u}^{(k+1)}, \boldsymbol{\sigma}^{(k+1)}\right)$ such that

$$
\left\{\begin{array}{l}
a\left(\boldsymbol{u}^{(k+1)}, \boldsymbol{v}\right)-\int_{\Gamma_{C}} \boldsymbol{\sigma}_{t}^{(k+1)} \cdot \boldsymbol{v}_{t} d s_{x}=L(\boldsymbol{v}) \quad \forall \boldsymbol{v} \in \boldsymbol{V}, \\
\boldsymbol{\sigma}_{t}^{(k+1)}+\rho_{k} \boldsymbol{u}_{t}^{(k+1)}=\boldsymbol{\sigma}_{t}^{(k)}+\rho_{k} \boldsymbol{u}_{t}^{(k)}-\omega \boldsymbol{R}_{\rho_{k}}\left(\boldsymbol{u}_{t}^{(k)}, \boldsymbol{\sigma}_{t}^{(k)}\right) \quad \text { on } \Gamma_{C} .
\end{array}\right.
$$

Step 3: Use a self-adaptive rule to update the parameter $\rho_{k+1}$ satisfying

$$
\frac{1}{1+s_{k}} \rho_{k} \leq \rho_{k+1} \leq\left(1+s_{k}\right) \rho_{k}
$$

Step 4: Stop if some given stopping criterion is satisfied, else set $k:=k+1$ and go to Step 1.

Let $\boldsymbol{u}^{*}$ and $\boldsymbol{\sigma}^{*}$ denote the solution of the frictional contact problem and the corresponding contact traction on the boundary $\Gamma$, respectively. In order to establish the convergence analysis of the projection method, we have to consider preliminary results presented in the form of two lemmas.

Lemma 3.1 If the sequence $\left\{s_{k}\right\}$ satisfies $s_{k} \geq 0$ and $\sum_{k=0}^{+\infty} s_{k}<+\infty$, then $\prod_{k=0}^{+\infty}\left(1+s_{k}\right)<+\infty$.

Lemma 3.2 Let $\left(\mathbf{u}^{(k)}, \sigma^{(k)}\right)$ be the sequence generated by the self-adaptive projection method, we have

$$
\begin{aligned}
& \left\|\boldsymbol{\sigma}_{t}^{(k+1)}-\boldsymbol{\sigma}_{t}^{*}+\rho_{k}\left(\boldsymbol{u}_{t}^{(k+1)}-\boldsymbol{u}_{t}^{*}\right)\right\|_{\Gamma_{C}}^{2} \\
& \quad \leq\left\|\boldsymbol{\sigma}_{t}^{(k)}-\boldsymbol{\sigma}_{t}^{*}+\rho_{k}\left(\boldsymbol{u}_{t}^{(k)}-\boldsymbol{u}_{t}^{*}\right)\right\|_{\Gamma_{C}}^{2}-2 \omega \rho_{k} \int_{\Gamma_{C}}\left(\boldsymbol{\sigma}_{t}^{(k)}-\boldsymbol{\sigma}_{t}^{*}\right) \cdot\left(\boldsymbol{u}_{t}^{(k)}-\boldsymbol{u}_{t}^{*}\right) d s_{x} .
\end{aligned}
$$

Lemma 3.1 is obvious and Lemma 3.2 can refer to Theorem 3.3 in [10]. We donate

$$
C_{s}:=\prod_{k=0}^{+\infty}\left(1+s_{k}\right)
$$

Consequently, it follows from Step 3 in Algorithm 1 that the parameter $\rho_{k} \in\left[\frac{1}{C_{s}} \rho_{0}, C_{s} \rho_{0}\right]$ is bounded. Let

$$
\rho_{L}:=\inf \left\{\rho_{k}\right\}_{k=0}^{+\infty}, \quad \rho_{U}:=\sup \left\{\rho_{k}\right\}_{k=0}^{+\infty}
$$

Then, we can prove the following convergence theorem.

Theorem 3.1 Let $\left\{\left(\boldsymbol{u}^{(k)}, \boldsymbol{\sigma}^{(k)}\right)\right\}$ be the sequence generated by the self-adaptive projection method, then $\mathbf{u}^{(k)}$ converges to $\mathbf{u}^{*}$ in $\boldsymbol{V}$ and $\boldsymbol{\sigma}^{(k)}$ converges to $\boldsymbol{\sigma}^{*}$ in $\left(L^{2}(\Gamma)\right)^{2}$.

Proof Note that $\boldsymbol{u}^{(k)}$ and $\boldsymbol{u}^{*}$ satisfy the same boundary conditions on $\Gamma_{D}$ and $\Gamma_{N}$. We apply the Green formula to (2.1)-(2.6) and use $u_{n}^{(k)}=u_{n}^{*}=0$, it follows that

$$
\int_{\Gamma_{C}}\left(\boldsymbol{\sigma}^{(k)}-\boldsymbol{\sigma}^{*}\right) \cdot\left(\boldsymbol{u}^{(k)}-\boldsymbol{u}^{*}\right) d s_{x}
$$




$$
\begin{aligned}
& =\int_{\Gamma_{C}}\left(\boldsymbol{\sigma}_{t}^{(k)}-\boldsymbol{\sigma}_{t}^{*}\right) \cdot\left(\mathbf{u}_{t}^{(k)}-\mathbf{u}_{t}^{*}\right) d s_{x} \\
& =a\left(\mathbf{u}^{(k)}-\mathbf{u}^{*}, \mathbf{u}^{(k)}-\mathbf{u}^{*}\right) \geq 0 .
\end{aligned}
$$

Since $0<\rho_{k+1} \leq\left(1+s_{k}\right) \rho_{k}$, we use (3.5) and (3.6) and have

$$
\begin{aligned}
\left\|\boldsymbol{\sigma}_{t}^{(k+1)}-\boldsymbol{\sigma}_{t}^{*}+\rho_{k+1}\left(\boldsymbol{u}_{t}^{(k+1)}-\boldsymbol{u}_{t}^{*}\right)\right\|_{\Gamma_{C}}^{2} \\
\leq\left(1+s_{k}\right)^{2}\left\|\boldsymbol{\sigma}_{t}^{(k+1)}-\boldsymbol{\sigma}_{t}^{*}+\rho_{k}\left(\boldsymbol{u}_{t}^{(k+1)}-\boldsymbol{u}_{t}^{*}\right)\right\|_{\Gamma_{C}}^{2} \\
\leq\left(1+s_{k}\right)^{2}\left\|\boldsymbol{\sigma}_{t}^{(k)}-\boldsymbol{\sigma}_{t}^{*}+\rho_{k}\left(\boldsymbol{u}_{t}^{(k)}-\boldsymbol{u}_{t}^{*}\right)\right\|_{\Gamma_{C}}^{2} \\
\quad-2 \omega \rho_{k}\left(1+s_{k}\right)^{2} \int_{\Gamma_{C}}\left(\boldsymbol{\sigma}_{t}^{(k)}-\boldsymbol{\sigma}_{t}^{*}\right) \cdot\left(\boldsymbol{u}_{t}^{(k)}-\boldsymbol{u}_{t}^{*}\right) d s_{x} \\
\leq\left(1+s_{k}\right)^{2}\left\|\boldsymbol{\sigma}_{t}^{(k)}-\boldsymbol{\sigma}_{t}^{*}+\rho_{k}\left(\boldsymbol{u}_{t}^{(k)}-\boldsymbol{u}_{t}^{*}\right)\right\| \\
\quad-2 \omega \rho_{k} \int_{\Gamma_{C}}\left(\boldsymbol{\sigma}_{t}^{(k)}-\boldsymbol{\sigma}_{t}^{*}\right) \cdot\left(\boldsymbol{u}_{t}^{(k)}-\boldsymbol{u}_{t}^{*}\right) d s_{x} .
\end{aligned}
$$

Then the following estimation is obtained by using $\xi_{k}:=2 s_{k}+s_{k}^{2}$ :

$$
\begin{aligned}
& \left\|\boldsymbol{\sigma}_{t}^{(k+1)}-\boldsymbol{\sigma}_{t}^{*}+\rho_{k+1}\left(\boldsymbol{u}_{t}^{(k+1)}-\boldsymbol{u}_{t}^{*}\right)\right\|_{\Gamma_{C}}^{2} \\
& \quad \leq\left(1+\xi_{k}\right)\left\|\boldsymbol{\sigma}_{t}^{(k)}-\boldsymbol{\sigma}_{t}^{*}+\rho_{k}\left(\boldsymbol{u}_{t}^{(k)}-\boldsymbol{u}_{t}^{*}\right)\right\|_{\Gamma_{C}}^{2} \\
& \quad-2 \omega \rho_{k} \int_{\Gamma_{C}}\left(\boldsymbol{\sigma}_{t}^{(k)}-\boldsymbol{\sigma}_{t}^{*}\right) \cdot\left(\boldsymbol{u}_{t}^{(k)}-\boldsymbol{u}_{t}^{*}\right) d s_{x} .
\end{aligned}
$$

From Lemma 3.1, we have $\sum_{k=0}^{+\infty} \xi_{k}<+\infty$ and $\prod_{k=0}^{+\infty}\left(1+\xi_{k}\right)<+\infty$. We introduce the following notations:

$$
\begin{array}{ll}
C_{0}:=\sum_{k=0}^{+\infty} \xi_{k}, & C_{p}:=\prod_{k=0}^{+\infty}\left(1+\xi_{k}\right), \\
\boldsymbol{\delta}_{\boldsymbol{u}}^{(k)}:=\boldsymbol{u}^{(k)}-\boldsymbol{u}^{*}, & \boldsymbol{\delta}_{\boldsymbol{u}_{t}^{(k)}}:=\boldsymbol{u}_{t}^{(k)}-\boldsymbol{u}_{t}^{*}, \\
\boldsymbol{\delta}_{\boldsymbol{\sigma}}^{(k)}:=\boldsymbol{\sigma}^{(k)}-\boldsymbol{\sigma}^{*}, & \boldsymbol{\delta}_{\boldsymbol{\sigma}_{t}}^{(k)}:=\boldsymbol{\sigma}_{t}^{(k)}-\boldsymbol{\sigma}_{t}^{*} .
\end{array}
$$

Then $\boldsymbol{\delta}_{\boldsymbol{u}}^{(k)}, \boldsymbol{\delta}_{\boldsymbol{u}_{t}}^{(k)} \in \boldsymbol{V}$ and $\boldsymbol{\delta}_{\boldsymbol{\sigma}}^{(k)}, \boldsymbol{\delta}_{\boldsymbol{\sigma}_{t}}^{(k)} \in\left(L^{2}\left(\Gamma_{C}\right)\right)^{2}$. From (3.5) we have

$$
\int_{\Gamma_{C}} \delta_{\sigma_{t}}^{(k)} \cdot \delta_{\mathbf{u}_{t}}^{(k)} d s_{x}=a\left(\delta_{\mathbf{u}}^{(k)}, \delta_{\mathbf{u}}^{(k)}\right) \geq \alpha\left\|\delta_{\mathbf{u}}^{(k)}\right\|_{\boldsymbol{v}}^{2}
$$

where $\alpha>0$. Combining (3.7) and (3.8) we get

$$
\begin{aligned}
\left\|\boldsymbol{\delta}_{\sigma_{t}}^{(k+1)}+\rho_{k+1} \boldsymbol{\delta}_{\mathbf{u}_{t}}^{(k+1)}\right\|_{\Gamma_{C}}^{2} & \leq\left(1+\xi_{k}\right)\left\|\boldsymbol{\delta}_{\sigma_{t}}^{(k)}+\rho_{2}^{(k)} \boldsymbol{\delta}_{\mathbf{u}_{t}}^{(k)}\right\|_{\Gamma_{C}}^{2} \\
& \leq \prod_{i=0}^{k}\left(1+\xi_{i}\right)\left\|\boldsymbol{\delta}_{\sigma_{t}}^{(0)}+\rho_{0} \boldsymbol{\delta}_{\mathbf{u}_{t}}^{(0)}\right\|_{\Gamma_{C}}^{2} \\
& \leq C_{p}\left\|\boldsymbol{\delta}_{\sigma_{t}}^{(0)}+\rho_{0} \boldsymbol{\delta}_{\boldsymbol{u}_{t}}^{(0)}\right\|_{\Gamma_{C}}^{2} .
\end{aligned}
$$


Therefore, there exists a constant $C>0$ such that

$$
\left\|\boldsymbol{\delta}_{\sigma_{t}}^{(k)}+\rho_{k} \boldsymbol{\delta}_{\mathbf{u}_{t}}^{(k)}\right\|_{\Gamma_{C}}^{2} \leq C, \quad \forall k \geq 0
$$

Consequently, both sequences $\left\{\boldsymbol{u}_{t}^{(k)}\right\}$ and $\left\{\boldsymbol{\sigma}_{t}^{(k)}\right\}$ are bounded. From (3.7), we also have

$$
\begin{aligned}
& 2 \omega \sum_{k=0}^{+\infty} \rho_{k} \int_{\Gamma_{C}}\left(\boldsymbol{\sigma}_{t}^{(k)}-\boldsymbol{\sigma}_{t}^{*}\right) \cdot\left(\boldsymbol{u}_{t}^{(k)}-\boldsymbol{u}_{t}^{*}\right) d s_{x} \\
& \quad \leq\left\|\boldsymbol{\delta}_{\sigma_{t}}^{(0)}+\rho_{0} \boldsymbol{\delta}_{\mathbf{u}_{t}}^{0)}\right\|_{\Gamma_{C}}^{2}+\sum_{k=0}^{+\infty} \xi_{k}\left\|\boldsymbol{\delta}_{\sigma_{t}}^{(k)}+\rho_{k} \boldsymbol{\delta}_{\mathbf{u}_{t}}^{(k)}\right\|_{\Gamma_{C}}^{2}
\end{aligned}
$$

We use (3.8), (3.9), and (3.10) and obtain

$$
\begin{aligned}
& \sum_{k=0}^{\infty}\left\|\delta_{\mathbf{u}}^{(k)}\right\|_{\boldsymbol{V}}^{2} \\
& \quad \leq\left(2 \omega \alpha \rho_{L}\right)^{-1}\left(\left\|\boldsymbol{\delta}_{\sigma_{t}}^{(0)}+\rho_{0} \boldsymbol{\delta}_{\mathbf{u}_{t}}^{0)}\right\|_{\Gamma_{C}}^{2}+\sum_{k=0}^{+\infty} \xi_{k}\left\|\boldsymbol{\delta}_{\sigma_{t}}^{(k)}+\rho_{k} \boldsymbol{\delta}_{\mathbf{u}_{t}}^{(k)}\right\|_{\Gamma_{C}}^{2}\right. \\
& \quad \leq\left(2 \omega \alpha \rho_{L}\right)^{-1}\left(C+C \sum_{k=0}^{+\infty} \xi_{k}\right) \\
& \quad \leq\left(2 \omega \alpha \rho_{L}\right)^{-1}\left(1+C_{0}\right) C .
\end{aligned}
$$

Hence, we have

$$
\lim _{k \rightarrow \infty}\left\|\delta_{\mathbf{u}}^{(k)}\right\|_{\boldsymbol{v}}^{2}=0
$$

which means that $\boldsymbol{u}^{(k)}$ converges to $\boldsymbol{u}^{*}$ in $\boldsymbol{V}$. From Step 2 of Algorithm 1 and Lebesgue's bounded convergence theorem it follows that $\sigma^{(k)}$ converges to $\sigma^{*}$ in $L^{2}\left(\Gamma_{C}\right)$ as $k \rightarrow \infty$.

\section{Implementation details of the proposed method}

Consider that our method generates a sequence of well-posed variational problems with the common boundary condition, we can easily obtain their numerical solutions. In this section, we describe the details of the method numerically.

\subsection{The self-adaptive rule}

Using the method developed in [9-13], we first suggest a simple rule to adjust the variable parameter $\rho_{k}$ for Step 3 of the self-adaptive projection method. Note that the sequence $\left\{\left(\boldsymbol{u}^{(k)}, \boldsymbol{\sigma}^{(k)}\right)\right\}$ generated by Algorithm 1 satisfies (3.5) and (3.8), we obtain

$$
\left\|\boldsymbol{\sigma}_{t}^{(k+1)}-\boldsymbol{\sigma}_{t}^{*}+\rho_{k}\left(\boldsymbol{u}_{t}^{(k+1)}-\boldsymbol{u}_{t}^{*}\right)\right\|_{\Gamma_{C}}^{2} \leq\left\|\boldsymbol{\sigma}_{t}^{(k)}-\boldsymbol{\sigma}_{t}^{*}+\rho_{k}\left(\boldsymbol{u}_{t}^{(k)}-\boldsymbol{u}_{t}^{*}\right)\right\|_{\Gamma_{C}}^{2}
$$

For the convergence speed of the method, we hope that

$$
\left\|\boldsymbol{\sigma}_{t}^{(k+1)}-\boldsymbol{\sigma}_{t}^{(k)}\right\|_{\Gamma_{C}}=\rho_{k}\left\|\boldsymbol{u}_{t}^{(k+1)}-\boldsymbol{u}_{t}^{(k)}\right\|_{\Gamma_{C}} .
$$


Using this idea, we update the parameter $\rho_{k}$ as follows. For a given positive constant $\tau$, if

$$
\rho_{k}\left\|\boldsymbol{u}_{t}^{(k+1)}-\boldsymbol{u}_{t}^{(k)}\right\|_{\Gamma_{C}}>(1+\tau)\left\|\boldsymbol{\sigma}_{t}^{(k+1)}-\boldsymbol{\sigma}_{t}^{(k)}\right\|_{\Gamma_{C}}
$$

we decrease $\rho_{k}$ in the next iteration; conversely, we increase $\rho_{k}$ when

$$
\rho_{k}\left\|\boldsymbol{u}_{t}^{(k+1)}-\boldsymbol{u}_{t}^{(k)}\right\|_{\Gamma_{C}}<\frac{1}{1+\tau}\left\|\boldsymbol{\sigma}_{t}^{(k+1)}-\boldsymbol{\sigma}_{t}^{(k)}\right\|_{\Gamma_{C}} .
$$

Let

$$
w_{k}=\frac{\left\|\boldsymbol{\sigma}_{t}^{(k+1)}-\boldsymbol{\sigma}_{t}^{(k)}\right\|_{\Gamma_{C}}}{\left\|\boldsymbol{u}_{t}^{(k+1)}-\boldsymbol{u}_{t}^{(k)}\right\|_{\Gamma_{C}}}
$$

we obtain the parameter $\rho_{k+1}$ according to the following self-adaptive rule:

$$
\rho_{k+1}= \begin{cases}\left(1+s_{k}\right) \rho_{k} & \text { if } w_{k}>(1+\tau) \rho_{k} \\ \frac{1}{1+s_{k}} \rho_{k} & \text { if } w_{k}<\frac{\rho_{k}}{1+\tau} \\ \rho_{k} & \text { otherwise }\end{cases}
$$

where the nonnegative sequence $\left\{s_{k}\right\}$ is generated by

$$
s_{k}= \begin{cases}\tau & \text { if } c_{k}<c_{k+1} \text { and } c_{k+1} \leq c_{\max } \\ \frac{1}{\left(c_{k+1}-c_{\max }\right)^{2}} & \text { if } c_{k}<c_{k+1} \text { and } c_{k+1}>c_{\max } \\ 0 & \text { otherwise }\end{cases}
$$

Let $c_{k}$ be the change times of $\left\{\rho_{k}\right\}$, i.e.,

$$
\mathrm{c}_{0}=0, \quad \mathrm{c}_{k+1}= \begin{cases}\mathrm{c}_{k} & \text { if } \frac{1}{1+\tau} \leq w_{k} \leq 1+\tau, \\ c_{k}+1 & \text { otherwise. }\end{cases}
$$

For a given constant integer $c_{\max }>0$, it follows that the sequence $\left\{s_{k}\right\}$ satisfies Lemma 3.1 automatically.

\subsection{The modified self-adaptive rule}

Following the above self-adaptive rule, we use the same way to choose $\rho_{k+1}$ when $w_{k}>(1+$ $\tau) \rho_{k}$ and $w_{k}<\frac{\rho_{k}}{1+\tau}$. Different from the self-adaptive rule for the case $\frac{\rho_{k}}{1+\tau} \leq w_{k} \leq(1+\tau) \rho_{k}$, we give a new rule to adjust $\rho_{k+1}$ as follows. From (4.1) we have

$$
\rho_{k}=\frac{\left\|\boldsymbol{\sigma}_{t}^{(k+1)}-\boldsymbol{\sigma}_{t}^{(k)}\right\|_{\Gamma_{C}}}{\left\|\boldsymbol{u}_{t}^{(k+1)}-\boldsymbol{u}_{t}^{(k)}\right\|_{\Gamma_{C}}}=w_{k},
$$

then we use $\rho_{k+1}=w_{k}$ to replace $\rho_{k+1}=\rho_{k}$. Consequently, we obtain the following modified self-adaptive rule:

$$
\rho_{k+1}= \begin{cases}\left(1+s_{k}\right) \rho_{k} & \text { if } w_{k}>(1+\tau) \rho_{k}, \\ \frac{1}{1+s_{k}} \rho_{k} & \text { if } w_{k}<\frac{\rho_{k}}{1+\tau}, \\ w_{k} & \text { otherwise. }\end{cases}
$$


Obviously, $\rho_{k+1}$ by using the modified self-adaptive rule also satisfies (3.4). Furthermore, this rule is more flexible to choose a proper parameter and improve the performance of the self-adaptive rule.

\subsection{Boundary element discrete form}

Problem (3.2)-(3.3) is the variational formulation of the following linear elasticity problem:

$$
\left\{\begin{array}{l}
\operatorname{div} \boldsymbol{\sigma}\left(\mathbf{u}^{(k+1)}\right)=\mathbf{0} \text { in } \Omega, \\
\mathbf{u}^{(k+1)}=\mathbf{0} \text { on } \Gamma_{D}, \\
T \mathbf{u}^{(k+1)}=\hat{\boldsymbol{t}} \text { on } \Gamma_{N}, \\
u_{n}^{(k+1)}=0 \text { on } \Gamma_{C}, \\
\boldsymbol{\sigma}_{t}^{(k+1)}+\rho_{k} \boldsymbol{u}_{t}^{(k+1)}=\boldsymbol{\sigma}_{t}^{(k)}+\rho_{k} \boldsymbol{u}_{t}^{(k)}-\omega \boldsymbol{R}_{\rho_{k}}\left(\boldsymbol{u}_{t}^{(k)}, \boldsymbol{\sigma}_{t}^{(k)}\right) \text { on } \Gamma_{C} .
\end{array}\right.
$$

Since the main iterative scheme (4.8) is in the boundary of the domain, the BEM is more appropriate for the methods $[9,10,17,18]$.

The solution $\boldsymbol{u}$ of problem (4.4)-(4.8) can be represented as the Betti-Somigliana representation formula

$$
\boldsymbol{u}(\boldsymbol{y})=\int_{\Gamma} \boldsymbol{G}(\boldsymbol{x}, \boldsymbol{y}) T \boldsymbol{u}(\boldsymbol{x}) d s_{\boldsymbol{X}}-\int_{\Gamma}\left(T_{\boldsymbol{x}} \boldsymbol{G}(\mathbf{x}, \boldsymbol{y})\right)^{T} \boldsymbol{u}(\boldsymbol{x}) d s_{\boldsymbol{x}},
$$

where the $\boldsymbol{x}$ in $T_{\boldsymbol{x}} \boldsymbol{G}(\boldsymbol{x}, \boldsymbol{y})$ denotes differentiations in (4.6) with respect to the variable $\boldsymbol{x}$, and $\boldsymbol{G}(\boldsymbol{x}, \boldsymbol{y})$ is the fundamental solution of the two-dimensional Lamé equation. The displacements $\boldsymbol{u}$ and tractions $T \boldsymbol{u}$ satisfy the boundary integral equation

$$
\frac{1}{2} \boldsymbol{u}(\boldsymbol{y})=\int_{\Gamma} \boldsymbol{G}(\boldsymbol{x}, \boldsymbol{y}) T \boldsymbol{u}(\boldsymbol{x}) d s_{\boldsymbol{X}}-\int_{\Gamma}\left(T_{\boldsymbol{x}} \boldsymbol{G}(\boldsymbol{x}, \boldsymbol{y})\right)^{T} \boldsymbol{u}(\boldsymbol{x}) d s_{\boldsymbol{X}} \quad \forall \boldsymbol{y} \in \Gamma
$$

The boundary $\Gamma$ is approximated by $N$ straight line elements. We use constant boundary elements, and the nodes are located in the middle of each element. Using functions $\boldsymbol{u}_{t}^{(k)}$ and $\boldsymbol{\sigma}_{t}^{(k)}$, we obtain the projection residual function $\boldsymbol{R}_{\rho_{k}}\left(\boldsymbol{u}_{t}^{(k)}, \boldsymbol{\sigma}_{t}^{(k)}\right)$ point-wise on nodes element by element along $\Gamma_{C}$. We apply $u_{n}=0$ and the Robin boundary condition

$$
\boldsymbol{\sigma}_{t}^{(k+1)}=\boldsymbol{\sigma}_{t}^{(k)}+\rho\left(\boldsymbol{u}_{t}^{(k)}-\boldsymbol{u}_{t}^{(k+1)}\right)-\omega \boldsymbol{R}_{\rho_{k}}\left(\boldsymbol{u}_{t}^{(k)}, \boldsymbol{\sigma}_{t}^{(k)}\right)
$$

to (4.10) to eliminate $\sigma_{n}^{(k+1)}$ and obtain the corresponding discrete form at $\boldsymbol{x} \in \Gamma_{C}$

$$
\begin{aligned}
& \frac{\boldsymbol{u}^{(k+1)}\left(\boldsymbol{y}_{i}\right)}{2}+\int_{\Gamma_{C}} \boldsymbol{u}^{(k+1)}\left(\boldsymbol{y}_{j}\right) T_{\boldsymbol{x}} \boldsymbol{G}\left(\boldsymbol{x}, \boldsymbol{y}_{i}\right)^{T} d s_{\boldsymbol{X}}+\int_{\Gamma_{C}} \rho_{k} \boldsymbol{u}_{t}^{(k+1)}\left(\boldsymbol{x}_{j}\right) \boldsymbol{G}\left(\boldsymbol{x}, \boldsymbol{y}_{i}\right) d s_{\boldsymbol{X}} \\
& =\int_{\Gamma_{C}}\left[\boldsymbol{\sigma}_{t}^{(k)}\left(\boldsymbol{x}_{j}\right)+\rho_{k} \boldsymbol{u}_{t}^{(k)}\left(\boldsymbol{x}_{j}\right)-\omega \boldsymbol{R}_{\rho_{k}}\left(\boldsymbol{u}_{t}^{(k)}\left(\boldsymbol{x}_{j}\right), \boldsymbol{\sigma}_{t}^{(k)}\left(\boldsymbol{x}_{j}\right)\right)\right] \boldsymbol{G}\left(\boldsymbol{x}, \boldsymbol{y}_{i}\right) d s_{\boldsymbol{x}} \quad \boldsymbol{y}_{i} \in \Gamma .
\end{aligned}
$$

On the boundary $\Gamma_{D} \cup \Gamma_{N}$, the discrete form of the boundary integral equation reads as follows:

$$
\begin{gathered}
\left.\frac{\mathbf{u}^{(k+1)}\left(\boldsymbol{y}_{i}\right)}{2}+\int_{\Gamma_{D} \cup \Gamma_{N}} \boldsymbol{u}^{(k+1)}\left(\mathbf{x}_{j}\right) T_{\boldsymbol{x}} \boldsymbol{G}(\mathbf{x}, \boldsymbol{y})\right)^{T} d s_{\boldsymbol{x}} \\
=\int_{\Gamma_{D} \cup \Gamma_{N}} T \mathbf{u}^{(k+1)}\left(\mathbf{x}_{j}\right) \mathbf{G}\left(\mathbf{x}, \mathbf{y}_{\mathbf{i}}\right) d s_{\mathbf{x}} \quad \boldsymbol{y}_{i} \in \Gamma .
\end{gathered}
$$


Using all the boundary conditions (4.5)-(4.8), we can solve the linear systems (4.12) and (4.13) to obtain both displacement $\boldsymbol{u}^{(k+1)}$ and stress $\boldsymbol{\sigma}^{(k+1)}$ at the nodes on $\Gamma$. So that we can reset the parameter and the Robin boundary condition on $\Gamma_{C}$ according to the method.

\section{Numerical experiments}

In this section, we present some numerical results to compare the performance of the projection method with different self-adaptive rules. In all tests, we take $\omega=1.8$ and $\tau=2$, $c_{\max }=10$ for our methods. Here, we choose $\frac{\left\|\mathbf{u}^{(k+1)}-\mathbf{u}^{(k)}\right\|_{L^{2}\left(\Gamma_{C}\right)^{+}+\left\|\boldsymbol{\sigma}_{t}^{(k+1)}-\sigma_{t}^{(k)}\right\|_{L^{2}\left(\Gamma_{C}\right)}}}{\left\|\mathbf{u}^{(k+1)}\right\|_{L^{2}\left(\Gamma_{C}\right)}+\left\|\boldsymbol{\sigma}_{t}^{(k+1)}\right\|_{L^{2}\left(\Gamma_{C}\right)}} \leq 10^{-6}$ as a stopping criterion and use Matlab codes for our methods.

\subsection{Example 1}

We consider an elastic body $\Omega=(0,8) \times(0,4)$. The bottom of the rectangle is in contact with a rigid foundation and the frictional contact part of the boundary is $\Gamma_{C}=(0,8) \times\{0\}$. On the part $\Gamma_{D}=(0,8) \times\{4\}$ the body is clamped. The Neumann boundary condition is given by $\hat{\boldsymbol{t}}=(400,0)^{\top}$ on $\{0\} \times(0,4)$, and $\hat{\mathbf{t}}=\mathbf{0}$ on the rest of $\Gamma_{N}$. The elasticity parameters are chosen to be Young's modulus $E=10,000$ and Poisson's ratio $v=0.3$, the friction coefficient is $g=150 \mathrm{daN} / \mathrm{mm}^{2}$ on $\Gamma_{C}$.

First we apply our methods to this problem with initial parameter $\rho=1$ and step size $h=0.05$, and the initial and deformed configurations of the body are shown in Fig. 1. On $\Gamma_{C}$, the tangential displacement and traction are depicted in Figs. 2 and 3, respectively. We note that $\Gamma_{C}$ is divided into a slip part and a stick part with a transition point from slip to stick near $(3.3,0)$. It can be seen that our results are in a good agreement with those in Ref. [5].

Here, we test the problem with different initial positive parameters $\rho$ and mesh sizes $h$. In Table 1, we give the number of iterations for the convergence of the self-adaptive projection and modified self-adaptive projection methods with rules (4.2) and (4.3), respectively. According to these numerical results, as expected, all parameters $\rho$ do not have a significant effect on the number of iterations for each method. Moreover, the number of

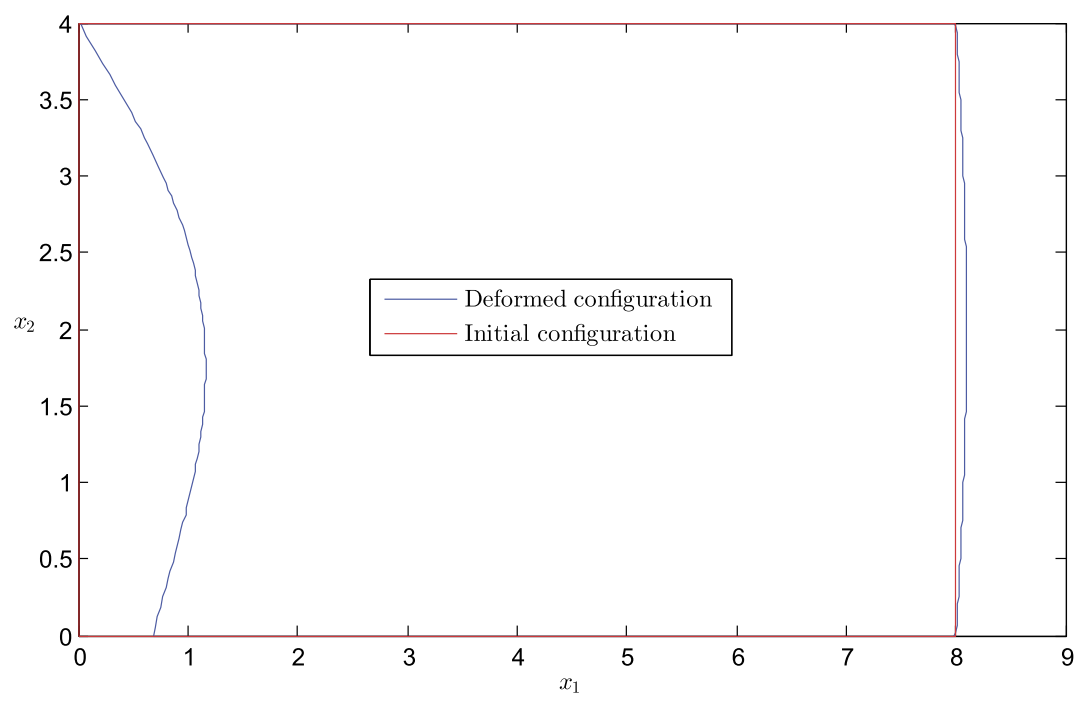

Figure 1 Initial and deformed configurations 


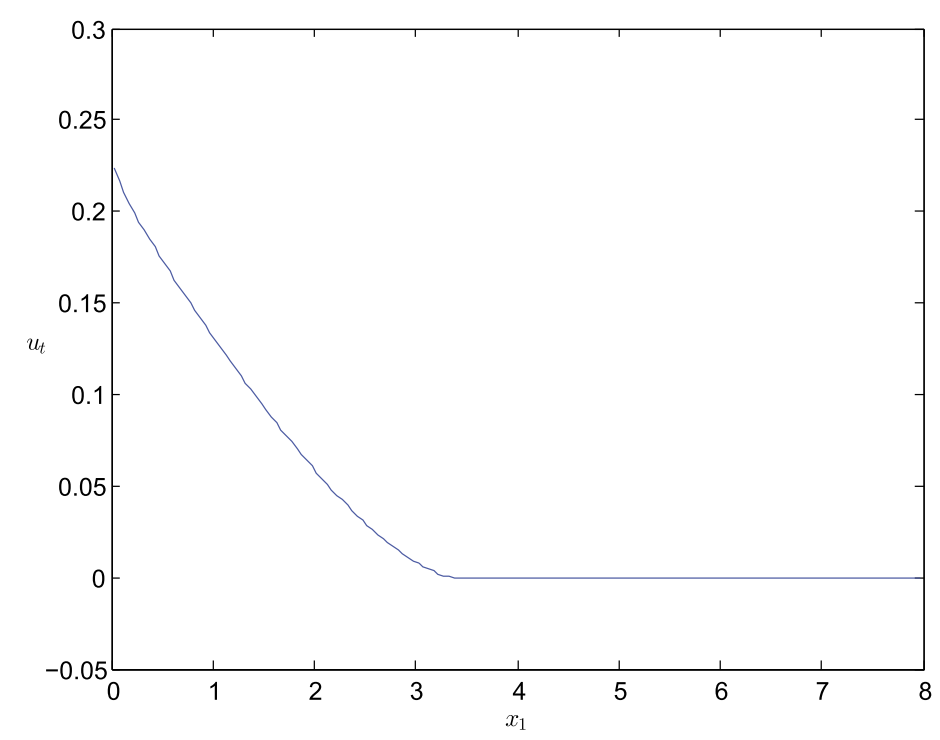

Figure 2 Tangential displacements on $\Gamma_{C}$

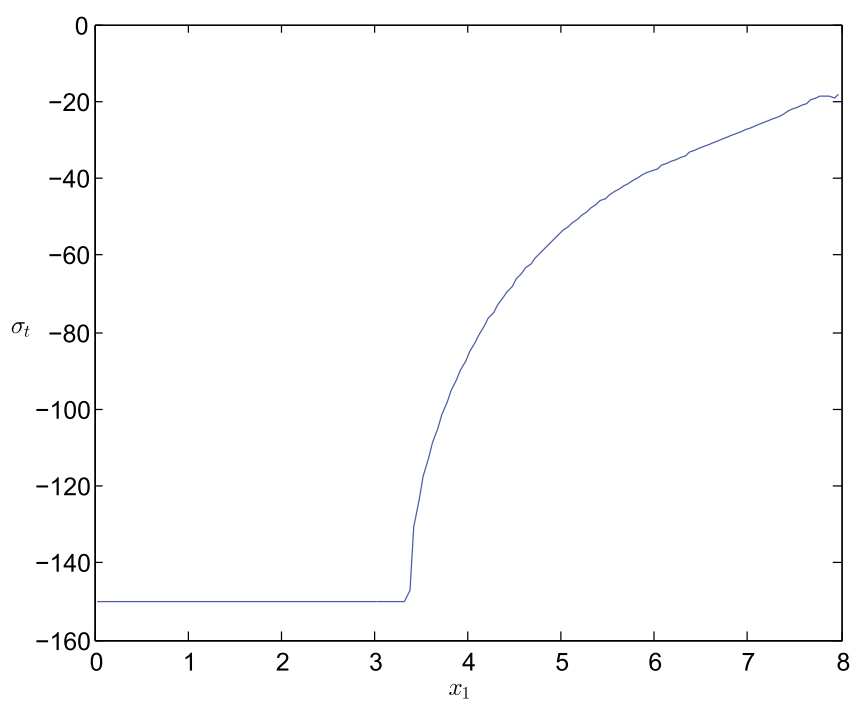

Figure 3 Tangential tractions on $\Gamma_{C}$

iterations for the method depends only weakly on $h$. In particular, the number of iterations of modified self-adaptive projection method is less than the self-adaptive projection method.

\subsection{Example 2}

In this example, we consider a frictional problem of the elastic square body $\Omega=(0,10) \times$ $(0,2)$ with $\Gamma_{C}=(0,10) \times\{0\}$. The Neumann boundary condition is given by $\hat{\boldsymbol{t}}=(1700,0)^{\top}$ on $\{0\} \times(0,2)$ and $\hat{\boldsymbol{t}}=(-1100,0)^{\top}$ on $\{10\} \times(0,2)$. And the homogeneous Dirichlet condition (i.e., $\mathbf{u}=\mathbf{0}$ ) is applied on $\Gamma_{D}:=(0,10) \times\{2\}$. The friction is $g=450 \mathrm{daN} / \mathrm{mm}^{2}$ on $\Gamma_{C}$. Young's modulus and Poisson's ratio are $E=2500$ and $v=0.2$, respectively. 
Table 1 Number of iterations for each method

\begin{tabular}{|c|c|c|c|c|c|c|c|c|}
\hline \multirow[t]{2}{*}{$\rho$} & \multicolumn{4}{|c|}{ Self-adaptive projection method } & \multicolumn{4}{|c|}{ Modified self-adaptive projection method } \\
\hline & $h=0.4$ & $h=0.2$ & $h=0.1$ & $h=0.05$ & $h=0.4$ & $h=0.2$ & $h=0.1$ & $h=0.05$ \\
\hline 1 & 59 & 81 & 70 & 78 & 46 & 51 & 55 & 62 \\
\hline 10 & 57 & 68 & 73 & 73 & 44 & 49 & 53 & 60 \\
\hline $10^{2}$ & 58 & 68 & 68 & 71 & 43 & 49 & 55 & 49 \\
\hline $10^{3}$ & 58 & 62 & 62 & 69 & 43 & 48 & 56 & 43 \\
\hline $10^{4}$ & 57 & 66 & 74 & 78 & 45 & 52 & 59 & 57 \\
\hline $10^{5}$ & 64 & 75 & 73 & 80 & 47 & 50 & 51 & 59 \\
\hline $10^{6}$ & 62 & 83 & 71 & 72 & 47 & 52 & 57 & 51 \\
\hline $10^{7}$ & 61 & 71 & 80 & 83 & 50 & 55 & 57 & 63 \\
\hline
\end{tabular}

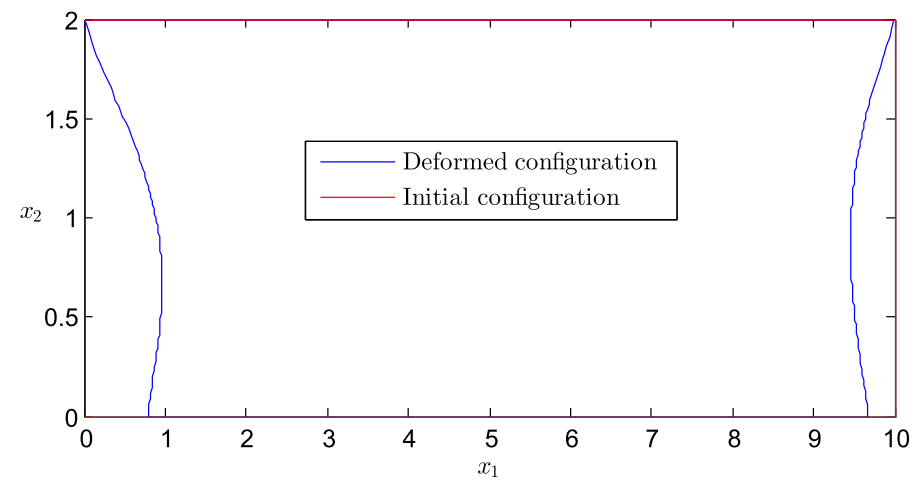

Figure 4 Initial and deformed configurations

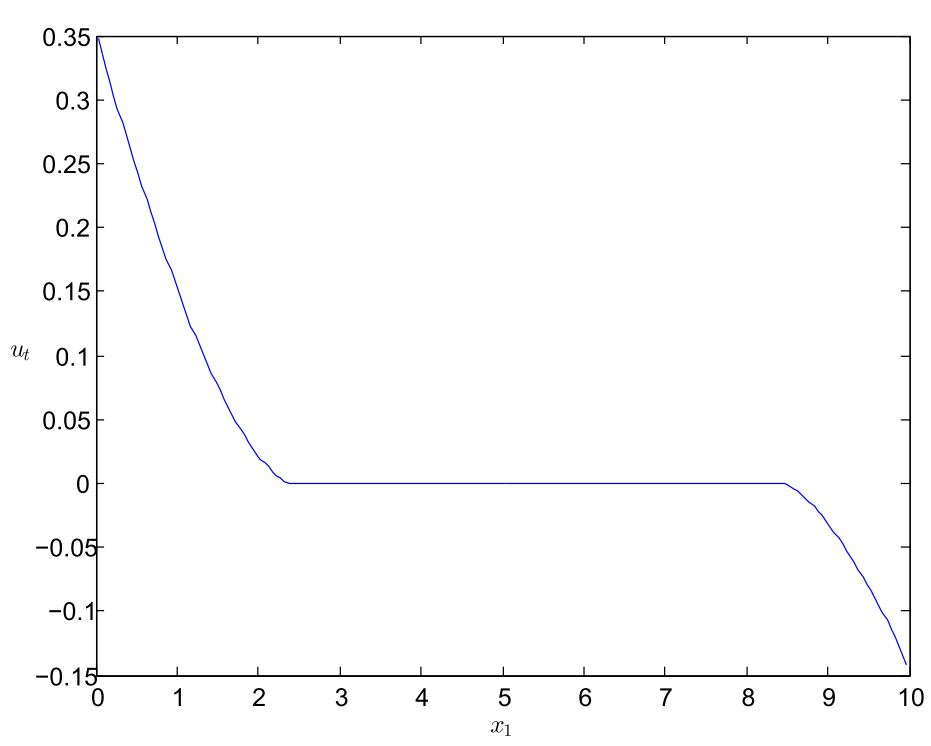

Figure 5 Tangential displacements on $\Gamma_{C}$

We choose $\rho=1$ and $h=0.05$ again and apply our method to this problem. Figure 4 depicts the initial and deformed configurations of the body, and Figs. 5 and 6 show the surface displacements and stresses on $\Gamma_{C}$. These numerical results show that $\Gamma_{C}$ is divided into two slip parts and a stick part with two transition points from slip to stick near 


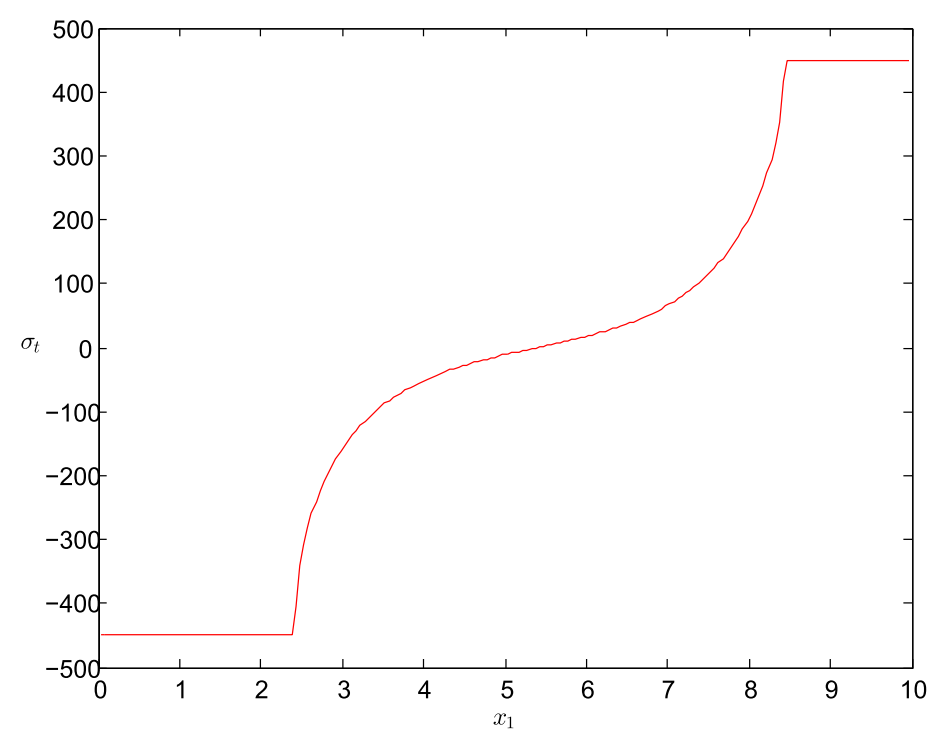

Figure 6 Tangential tractions on $\Gamma_{\mathrm{C}}$

Table 2 Number of iterations for each method

\begin{tabular}{|c|c|c|c|c|c|c|c|c|}
\hline \multirow[t]{2}{*}{$\rho$} & \multicolumn{4}{|c|}{ Self-adaptive projection method } & \multicolumn{4}{|c|}{ Modified self-adaptive projection method } \\
\hline & $h=0.4$ & $h=0.2$ & $h=0.1$ & $h=0.05$ & $h=0.4$ & $h=0.2$ & $h=0.1$ & $h=0.05$ \\
\hline 1 & 53 & 64 & 71 & 55 & 43 & 47 & 50 & 51 \\
\hline 10 & 49 & 62 & 72 & 62 & 42 & 45 & 48 & 38 \\
\hline $10^{2}$ & 50 & 55 & 59 & 55 & 40 & 44 & 47 & 49 \\
\hline $10^{3}$ & 47 & 58 & 64 & 57 & 39 & 42 & 45 & 46 \\
\hline $10^{4}$ & 49 & 59 & 71 & 63 & 41 & 44 & 48 & 48 \\
\hline $10^{5}$ & 49 & 57 & 61 & 54 & 42 & 44 & 46 & 48 \\
\hline $10^{6}$ & 53 & 63 & 70 & 54 & 45 & 46 & 49 & 49 \\
\hline $10^{7}$ & 54 & 65 & 74 & 67 & 46 & 48 & 52 & 54 \\
\hline
\end{tabular}

$(2.4,0)$ and $(8.4,0)$. It can be observed that our results are in agreement with conditions (2.5) and (2.6) again. We also investigate the convergence behavior of our method for this example. Table 2 displays the number of iterations with respect to the parameter $\rho$ and the mesh size $h$. As in the previous example, our modified self-adaptive projection method is better than the self-adaptive projection method, because this method accelerates the convergence speed.

\section{Conclusion}

This paper provides the analysis of two projection methods for the solution of frictional contact problems. Our methods show unconditional convergence and only require solving a sequence of general linear elasticity problems. In particular, we propose two adjustment rules to choose the proper parameter $\rho$ automatically. As the main iterative process is given on the boundary of the domain, the unknowns of the problem are computed easily by using the boundary element method. Furthermore, the numerical results show that the modified self-adaptive projection method has better performance than the self-adaptive projection method. Proposed methods can be extended to frictional contact problems in three space dimensions (3D). 
Acknowledgements

Not applicable.

\section{Funding}

This work is funded by the National Natural Science Foundation Project of CQ CSTC of China (Grant nos. cstc2017jcyjAX0316 and cstc2016jcyjA0419) and the School Fund Project of Chongqing Normal University (CQNU) (Grant no. 16XZH07).

\section{Availability of data and materials}

All data are fully available without restriction.

\section{Competing interests}

The authors declare that they have no competing interests.

\section{Authors' contributions}

All authors equally have made contributions. All authors read and approved the final manuscript.

\section{Author details}

${ }^{1}$ School of Mathematical Sciences, Chongqing Normal University, Chongqing, P.R. China. ${ }^{2}$ College of Computer and Information Science, Chongqing Normal University, Chongqing, P.R. China.

\section{Publisher's Note}

Springer Nature remains neutral with regard to jurisdictional claims in published maps and institutional affiliations.

\section{Received: 26 September 2018 Accepted: 7 April 2019 Published online: 15 April 2019}

\section{References}

1. Glowinski, R.: Numerical Methods for Nonlinear Variational Problems. Springer, Berlin (2008)

2. Han, W.M.: A Posteriori Error Analysis Via Duality Theory. Springer, New York (2005)

3. Stadler, G.: Semismooth Newton and augmented Lagrangian methods for a simplified friction problem. SIAM J. Optim. 15, 39-62 (2004)

4. De Los Reyes, J.C., Hintermüller, M.: A duality based semismooth Newton framework for solving variational inequalities of the second kind. Interfaces Free Bound. 13, 437-462 (2011)

5. Bostan, V., Han, W.M.: A posteriori error analysis for finite element solutions of a frictional contact problem. Comput. Methods Appl. Mech. Eng. 195, 1252-1274 (2006)

6. Denkowski, Z., Migórski, S., Ochal, A.: A class of optimal control problems for piezoelectric frictional contact models. Nonlinear Anal., Real World Appl. 12, 1883-1895 (2011)

7. Touzaline, A.: Optimal control of a frictional contact problem. Acta Math. Appl. Sin. Engl. Ser. 31, 991-1000 (2015)

8. Wang, F., Han, W.M., Cheng, X.L.: Discontinuous Galerkin methods for solving a quasistatic contact problem. Numer. Math. 126, 771-800 (2014)

9. Zhang, S.G., Li, X.L.: A self-adaptive projection method for contact problems with the BEM. Appl. Math. Model. 55, 145-159 (2018)

10. Zhang, S.G., Li, X.L., Ran, R.S.: Self-adaptive projection and boundary element methods for contact problems with Tresca friction. Commun. Nonlinear Sci. Numer. Simul. 68, 72-85 (2019)

11. He, B.S., Liao, L.Z., Wang, S.L.: Self-adaptive operator splitting methods for monotone variational inequalities. Numer Math. 94, 715-737 (2003)

12. Bnouhachem, A.: An inexact implicit method for general mixed variational inequalities. J. Comput. Appl. Math. 200 417-426 (2007)

13. Ge, Z.L., Han, D.R.: Self-adaptive implicit methods for monotone variant variational inequalities. J. Inequal. Appl. 2009, Article ID 458134 (2009)

14. Chouly, F: A Nitsche-based method for unilateral contact problems: numerical analysis. SIAM J. Numer. Anal. 51, 1295-1307 (2013)

15. Chouly, F:: An adaptation of Nitsche's method to the Tresca friction problem. J. Math. Anal. Appl. 411, 329-339 (2014)

16. Zhang, S.G., Li, X.L.: An augmented Lagrangian method for the Signorini boundary value problem with BEM. Bound. Value Probl. 2016, 62 (2016)

17. Hsiao, G.C., Wendland, W.L.: Boundary Integral Equations. Springer, Berlin (2008)

18. Steinbach, O.: Numerical Approximation Methods for Elliptic Boundary Value Problems. Springer, New York (2008) 